\title{
Ultrassonografia quantitativa do fígado em gatos com tirotoxicose induzida
}

Warley Gomes dos SANTOS ${ }^{1}$

Jessica Nascimento Moraes MONTEIRO $^{1}$

Daniel Capucho de OLIVEIRA $^{1}$

Daniel Cometti BORLINI Sebastião MARTINS FILHO ${ }^{2}$ Flávia Mara MACHADO 3 Louisiane de Carvalho NUNES ${ }^{1}$

Fabiano Séllos COSTA ${ }^{1}$

\section{Correspondência para:}

Warley Gomes dos Santos e/ou Fabiano Séllos Costa, Universidade Federal do Espírito Santo, Centro de Ciências Agrárias, Departamento de Medicina Veterinária, Alto Universitário $\mathrm{s} / \mathrm{n}^{\circ}$, Alegre, ES, Brasil, 29500-000, warleygomessantos@hotmail.com

Recebido para publicação: 02/06/2008 Aprovado para publicação: 27/08/2009

\author{
1 - Departamento de Medicina Veterinária da Universidade Federal do \\ Espírito Santo \\ 2 - Departamento de Informática: Área de estatística da Universidade Federal \\ de Viçosa \\ 3 - Curso de Medicina Veterinária do Centro Universitário Vila Velha
}

\section{Resumo}

Este estudo avaliou ultrassonograficamente o fígado de gatos submetidos à tirotoxicose induzida pela técnica de histograma dos níveis de cinza com o objetivo de melhor avaliar possíveis alterações hepáticas decorrentes do excesso de hormônios tiroidianos. Para isto, foram utilizados nove gatos hígidos, adulto-jovens, que receberam diariamente $150 \mu \mathrm{g} / \mathrm{kg}$ de levotiroxina sódica, por 60 dias. O histograma hepático não demonstrou diferença estatística ao nível de $5 \%$ de significância entre os momentos avaliados; entretanto, as variações dos valores das variáveis estudadas sugeriram a promoção de aumento da heterogeneidade e diminuição da ecogenicidade do parênquima hepático quando comparado ao momento inicial. A avaliação citopatológica e histopatológica revelou quadro de hepatite tirotóxica em todos os animais.

\section{Introdução}

O hipertiroidismo nos gatos é uma alteração clínica multissistêmica resultante de excessivas concentrações dos hormônios tiroidianos. Atualmente, de acordo com a literatura norte americana, esta é considerada a doença endócrina de maior freqüência nos gatos domésticos, acometendo principalmente animais senescentes. $^{1,2}$

Devido à crescente prevalência do hipertiroidismo nos gatos, são necessários estudos em vários sistemas orgânicos, sobretudo o fígado, por ser um órgão altamente responsivo à ação dos hormônios tiroidianos. O fígado atua ativamente no metabolismo central e dos hormônios tiroidianos, assim como níveis séricos adequados de tiroxina e triiodotironina são importantes para a preservação da função hepática normal. ${ }^{3}$ Os hormônios produzidos pela glândula tiróide são metabolizados principalmente pelo complexo enzimático das vias glicuronil-transferases, excretados pela vesícula biliar, e com significativa reassimilação dos metabólitos pela recirculação entero-hepática. ${ }^{4}$ Em particular os felinos apresentam dificuldade para metabolizar algumas substâncias pela via da conjugação glucoronil-transferase, entretanto, os efeitos da deficiência de glucuronidação depende da natureza da droga e da presença ou não de vias alternativas para biotransformação, mas em relação aos hormônios tiroidianos, a literatura relata que estes animais não têm grandes problemas. A via da sulfatação é uma via alternativa para o metabolismo, sendo bem desenvolvida nestes animais, e também auxilia na metabolização dos hormônios tiroidianos tornando os metabólitos mais polares, para facilitar a excreção. ${ }^{5}$

O excesso dos hormônios tiroidianos (tirotoxicose) é caracterizado pela manifestação de sinais e sintomas clínicos que podem variar de leves a graves. 
A gravidade da sintomatologia apresentada irá depender diretamente do estágio em que a doença se encontra e da coexistência de outras alterações sistêmicas. ${ }^{6}$ Podem ocorrer diversas alterações nas células do fígado decorrentes da tirotoxicose, tais como esteatose, lipidose, degeneração hidrópica, hiperplasia e hipertrofia dos hepatócitos, depleção de glicogênio nos hepatócitos, infiltrado mononuclear e polimorfonuclear, hiperplasia das células de Kupffer e necrose. Alterações crônicas no fígado podem estar presentes e variam de fibrose portal leve até cirrose e redução da glutationa, com aumento de radicais reativos de oxigênio. ${ }^{7,8,9}$

Nem sempre as alterações supracitadas são diagnosticadas pela ultrassonografia abdominal, não sendo possível descartar uma hepatopatia difusa quando o órgão apresenta-se normal ao exame, uma vez que se pode não ocorrer distorção da textura ou da arquitetura interna do parênquima. ${ }^{10,11} \mathrm{O}$ acúmulo de gordura promove maior reflexão das ondas sonoras elevando a ecogenicidade do parênquima hepático em relação ao ligamento falciforme. ${ }^{12,13}$ Já a degeneração hidrópica intracelular, como a que ocorre na hepatite aguda, diminui a ecogenicidade do fígado. ${ }^{14,15}$

A realização de exames citopatológicos e histopatológicos do fígado pode ser necessária para a conclusão do diagnóstico, sendo indicada a sua realização por via percutânea e com o auxílio do exame ultrassonográfico, visando melhor localizar o ponto de coleta e minimizar os riscos do procedimento. ${ }^{9}$

Outro fator que contribui para a crescente difusão da ultrassonografia abdominal como meio de investigação de afecções hepáticas é a possibilidade de avaliar a dimensão, forma, contornos, bordos, penetração dos feixes e arquitetura interna do órgão. Apesar dos inúmeros benefícios, o exame ultrassonográfico pode apresentar divergência na interpretação dos seus achados, devido à análise subjetiva e individual. Uma avaliação comparativa entre os órgãos abdominais é importante para melhor caracterização de possíveis alterações ultrassonográficas. Os padrões de normalidade demonstram que a ecotextura do parênquima hepático é homogênea, sendo levemente mais grosseira que o baço. A ecogenicidade do fígado de gatos normais é igual ou discretamente maior em relação ao córtex renal, ligeiramente menor quando comparado ao parênquima esplênico e variando de isoecogênico a discretamente hipoecogênico quanto ao ligamento falciforme. ${ }^{16}$ Vale ressaltar que gatos clinicamente normais, mas obesos, podem ter um fígado que é mais hiperecogênico que o ligamento falciforme. ${ }^{13}$

Devido ao caráter subjetivo do exame ultrassonográfico podem-se gerar divergências para a interpretação de seus achados, no qual diferentes fatores podem influenciar no estabelecimento de um diagnóstico, tais como a experiência do avaliador, aparelho utilizado e regulagem de profundidade, ganho e contraste. ${ }^{15,16}$ Concomitantemente às alterações que ocorrem no fígado, diversas enfermidades podem promover alterações em outros órgãos usados para a comparação da ecogenicidade e ecotextura, sendo este mais um fator que pode dificultar o diagnóstico. ${ }^{15}$ Para tentar evitar possíveis erros de interpretação e minimizar a subjetividade do exame, podemos utilizar técnicas que quantificam a ecogenicidade e a ecotextura das regiões avaliadas. ${ }^{11,17,18,19,20}$

O método do histograma possibilita uma avaliação quantitativa da ecotextura e ecogenicidade de uma região previamente selecionada, demonstrando a distribuição de freqüência na intensidade do eco na região de interesse e mensurando a quantidade de níveis de cinza. O aparelho de ultrassonografia gera um gráfico do tipo histograma, demonstrando neste toda a variação dos 
espectros de cinza que compõem a imagem da área selecionada. A imagem ultrassonográfica é gerada em tons de cinza. Assim é formada pelo preto absoluto, com valor zero, passando por muitas tonalidades de cinza até o branco absoluto, valor 255. A função do histograma dos níveis de cinza quantifica todas as tonalidades presentes na região de interesse selecionada. Esta forma de análise está disponível em muitos aparelhos ultra-sonográficos comerciais, entretanto sua aplicação tem sido restrita ao meio acadêmico, merecendo padronização de valores para maior uso na prática clínica e em protocolos experimentais na medicina humana e veterinária. ${ }^{19}$

A reposição hormonal em excesso, em humanos com hipotiroidismo, pode provocar um quadro hipermetabólico iatrogênico idêntico ao observado na tirotoxicose endógena. Este fato assume importância, uma vez que os hormônios da tireóide estão entre as medicações mais prescritas na medicina humana. ${ }^{21}$

Para melhor caracterização do hipertiroidismo felino foram realizados protocolos experimentais de indução com a suplementação diária de levotiroxina sódica, promovendo uma elevação significativa dos níveis séricos dos hormônios tiroidianos, similar ao observado na tirotoxicose de origem endógena. Estudos experimentais com indução a um estado de tirotoxicose foram desenvolvidos em cães ${ }^{22}$; $\operatorname{ratos}^{8,23,24,24,26}$; em felinos domésticos ${ }^{27,28,29,30}$ e humanos ${ }^{31}$, sendo possível a demonstração de alterações metabólicas similares ao hipertiroidismo endógeno. Desta forma, o objetivo deste experimento é demonstrar por meio da ultrassonografia quantitativa as alterações hepáticas da tirotoxicose induzida em felinos domésticos.

\section{Material e Método}

Este estudo foi realizado sob aprovação do comitê de ética e proteção animal da FMVZ/UNESP-Botucatu, protocolo 49/2002 - CEEA, sendo respeitados todos os preceitos éticos de proteção aos animais. Para a realização deste protocolo, foram utilizados nove gatos domésticos, adulto-jovens, sem distinção de sexo ou raça e com peso médio de 2,8 $\pm 0,5 \mathrm{~kg}$. Todos os animais do grupo experimental foram clinicamente avaliados, e foi realizada a dosagem sérica de proteínas totais, uréia e das enzimas ALT, AST e FA, sendo utilizados somente os animais que se apresentam normais após a realização desses exames de triagem.

Reiteramos que não foi encontrado na literatura consultada algum trabalho que tivesse avaliado quantitativamente a ecogenicidade e ecotextura do fígado de gatos com tirotoxicose pela técnica do histograma dos níveis de cinza, sendo o objetivo de outro manuscrito de nossa autoria a padronização dos valores médios da ecogenicidade e ecotextura do fígado de gatos. Para a indução à tirotoxicose, administrou-se por via oral $150 \mu \mathrm{g} / \mathrm{kg}$ de levotiroxina sódica a cada 24 horas durante 60 dias, conforme protocolo executado anteriormente por outros autores $\mathrm{s}^{9,27,28,29,30}$, com doses reajustadas conforme a variação de peso dos animais durante o período experimental.

Para a realização do histograma dos níveis de cinza do fígado, mensurações quantitativas foram avaliadas pelas variáveis (NMOST/NALL) sendo NMOST referente a todos os pontos luminosos semelhantes e NALL os pontos luminosos totais presentes na região selecionada representando a relação NMOST/NALL a ecotextura da região selecionada. $\mathrm{O}$ desvio padrão da amplitude do eco "STANDARD DEVIATION" (SD) também constitui outro valor referente à ecotextura, pois ele mensura a amplitude que o eco variou. Quanto menor os valores de SD mais homogênea é a imagem. A ecogenicidade é representada pela variável (LMEAN), que indica a luminosidade média da região 
selecionada. Médias baixas do LMEAN indicam imagens mais escuras, e médias altas, corresponde a imagens mais claras. $\mathrm{O}$ eixo $\mathrm{X}$ do gráfico do histograma representa os níveis de cinza, e o eixo $\mathrm{Y}$ a quantidade de pixel para cada nível de cinza. ${ }^{32}$

Anteriormente à realização da avaliação ultrassonográfica hepática foi realizada tricotomia da região abdominal de cada animal com posterior aplicação de gel acústico. Estas análises quantitativas foram obtidas do parênquima hepático no momento inicial (M0) e após 60 dias do início do protocolo experimental (M1). O aparelho ultrassonográfico utilizado foi o modelo HS-2000 da Honda, com o transdutor linear na freqüência de $10 \mathrm{MHz}$, ganho 97. A medida do histograma foi obtida a partir da seleção de uma região de interesse quadrangular com área de $25 \mathrm{~mm}^{2}$ lateralmente à vesícula biliar e em uma profundidade oscilando entre 2 e $3 \mathrm{~cm}$.

Para a obtenção das amostras de fígado, realizada posteriormente ao exame ultrassonográfico, os felinos foram submetidos à anestesia dissociativa com cloridrato de tiletamina e zolazepam, por via intramuscular, sendo coletadas amostras citopatológicas por punção aspirativa por agulha fina e histopatológica, com agulhas tipo "TruCut", ambos por via percutânea e guiados por ultrassom. As amostras de exame citopatológicos foram distentidas sobre lâminas para a realização de esfregaço tipo "squash", fixadas em metanol por três a cinco minutos e corados pelo método de panótico rápido. As biópsias foram fixadas em solução de formalina a $10 \%$ e submetidas ao processamento de rotina pela inclusão em parafina. Cortes de cinco micrômetros de espessura foram feitos em micrótomo rotativo e o material em seguida, foi corado pelo método de hematoxilina e eosina. As lâminas para os exames citopatológicos e histopatológicos foram avaliadas com base nas alterações morfológicas observadas.

Para a análise estatística das variáveis Nmost/Nall, Lmean e SD, durante o período experimental, utilizou-se o teste não paramétrico de Wilcoxon a $5 \%$ de significância. Os exames citopatológicos e histopatológicos foram realizados com o intuito de verificar a acurácia do histograma dos níveis de cinza e foram analisados apenas de forma descritiva.

\section{Resultados, Discussão e Conclusões}

Outros autores ${ }^{9,27,28,29,30}$, procurando melhor esclarecer os aspectos fisiopatológicos nos felinos domésticos, realizaram protocolos de indução ao estado de tirotoxicose similar ao realizado em nosso experimento, promovendo uma elevação da concentração sérica dos hormônios tiroidianos compatíveis com os observados no hipertiroidismo espontâneo nesta espécie. Vale ressaltar, que apesar das pesquisas desenvolvidas na medicina humana e veterinária, não foi encontrado na literatura consultada trabalhos que tivessem avaliado quantitativamente a ecogenicidade e ecotextura do fígado de gatos com tirotoxicose pela técnica do histograma dos níveis de cinza. Deste modo, na literatura, não consta a padronização dos valores de normalidade para a ecogenicidade e ecotextura do fígado de gatos, sendo que no presente trabalho, os valores do momento inicial serviram como referência para as alterações ou achados após o desenvolvimento da tirotoxicose experimental.

Deve-se salientar que a tirotoxicose experimental em gatos apresenta algumas particularidades importantes que diferem dos casos espontâneos. ${ }^{9}$ Neste estudo foram utilizados animais adulto-jovens, que diferentemente no hipertiroidismo felino espontâneo, acomete gatos senis. Outro ponto é que o grupo experimental utilizado não apresentava alterações clínicas e/ou doenças concomitante, visto que no hipertiroidismo espontâneo os animais 
podem ter outras afecções de origem nãotiroideanas e mascarar os resultados do hipertiroidismo.

Assim como já previamente descrito para outros sistemas ${ }^{1,2,3}$, foi possível observar que um período relativamente curto de indução ao estado de tirotoxicose na espécie felina é capaz de promover alterações no parênquima hepático. Este fato reforça a importância de um diagnóstico precoce da doença e o estabelecimento imediato de uma terapia adequada, visando melhorar seu prognóstico. No homem, é freqüente a ocorrência de tirotoxicose iatrogênica em pacientes com hipotiroidismo. ${ }^{21}$ Por este motivo, salientamos a necessidade de se evitar suplementações excessivas dos hormônios tiroidianos nos casos em que esta medicação for indicada para gatos, e também extrapolemos para cães, pois é a espécie que é mais comum a utilização destes hormônios em quadros de hipotiroidismo.

A relação NMOST/NALL é um valor quantitativo que expressa a heterogeneidade ou homogeneidade do parênquima pelo aumento ou da redução respectivamente. Neste estudo não foi caracterizada diferença estatística que demonstre variação nesta relação (Tabela 1), entretanto, entre os nove animais estudados, sete apresentaram algum aumento dos valores de NMOST/ NALL, sugerindo uma maior heterogeneidade da região avaliada. Alguns autores relatam que nem sempre as hepatopatias difusas provocam mudanças significativas na ecotextura do órgão, sendo esta alteração mais freqüente na presença de lesões focais. ${ }^{11}$ Pode-se ter um exame ultrassonográfico normal, sem estar

Tabela 1 - Valores individuais e médios de NM/Nall,* LMEAN** e SD*** de nove gatos nos momentos inicial (M0), e após 8 semanas (M1) do protocolo de indução a tirotoxicose

\begin{tabular}{|c|c|c|c|c|c|c|}
\hline \multirow[t]{2}{*}{ Gato } & \multicolumn{2}{|c|}{ NMOST/NALL } & \multicolumn{2}{|c|}{ L MEAN } & \multicolumn{2}{|c|}{ SD } \\
\hline & Mo & Ml & Mo & Ml & Mo & Ml \\
\hline 1 & 23,5 & 32,2 & 6,3 & 4,7 & 14,2 & 10,17 \\
\hline 2 & 33,0 & 26,7 & 3,7 & 4,9 & 10,5 & 13,5 \\
\hline 3 & 24,0 & 35,0 & 6,5 & 5,8 & 13,2 & 8,55 \\
\hline 4 & 22,9 & 31,8 & 6,5 & 4,6 & 13,8 & 9.47 \\
\hline 5 & 27,4 & 30,9 & 5,0 & 5.7 & 10,5 & 11,05 \\
\hline 6 & 37,2 & 28,6 & 3,1 & 5,8 & 8,4 & 10,73 \\
\hline 7 & 29,0 & 32,3 & 6,1 & 5,2 & 11,8 & 11,25 \\
\hline 8 & 29,5 & 32,2 & 6,6 & 4,9 & 10,5 & 9,7 \\
\hline 9 & 27,9 & 32,4 & 7,2 & 5,3 & 11,3 & 11,9 \\
\hline Média & $28.3 \mathrm{a}^{1 /}$ & $31.3 \mathrm{a}$ & $5.7 \mathrm{a}$ & $5.2 \mathrm{a}$ & 11.6a & $10.7 \mathbf{a}$ \\
\hline
\end{tabular}


acompanhando por alterações da textura e arquitetura interna do parênquima. ${ }^{10}$

Zablith $^{9}$ demonstrou que em um período de 42 dias de tiroxicose induzida gerou-se um quadro de hepatite aguda, fato que foi comprovado pela diminuição subjetica da ecogenicidade hepática em aproximadamente $87 \%$ dos animais estudados ao exame ultrassonográfico e infiltrado periportal hiperecogênico em aproximadamente $62 \%$ dos animais variando de discreto a severo. Segundo a literatura ${ }^{12,14,15}$, uma diminuição difusa da ecogenicidade pode estar correlacionado a processos inflamatórios ou congestivos. Embora não tenha sido caracterizada diferença estatística para os valores de LMEAN, seis animais do grupo experimental $(66 \%)$ apresentaram menores valores para esta variável, conforme demonstrado na tabela 1 , indicando quantitativamente a diminuição da ecogenicidade do parênquima hepático na região selecionada. Utilizando-se de avaliação ultrassonográfica subjetiva, também foi observado por outro autor ${ }^{9}$ uma diminuição de ecogenicidade do parênquima hepático em gatos com tirotoxicose induzida, observando-se o padrão de "fígado em céu estrelado".

Um dos animais variou o LMEAN de 3,1 para 5,8 ao final do protocolo experimental e apresentou acentuada vacuolização citoplasmática no exame citopatológico, achado característico de esteatose hepática, uma vez que, de acordo com a literatura ${ }^{12,13}$, o acúmulo de gordura nos diferentes tecidos promove maior reflexão das ondas sonoras elevando a ecogenicidade do parênquima hepático em relação ao ligamento falciforme. Segundo Zablith ${ }^{9}$ é possível a ocorrência de acúmulo de gordura nos hepatócitos na ocorrência de hipertiroidismo, porém somente este animal apresentou achados ultrassonográficos e citopatológicos compatíveis com esta alteração.

O Standard Deviation (SD) pode representar a homogeneidade ou heterogeneidade do parênquima do órgão fornecendo dados referentes ao desvio padrão da amplitude do eco. O fígado com textura normal ou degeneração gordurosa, apresenta valores de SD com médias próximas, embora haja tendência ao aumento com a infiltração de lipídios e fibrose, havendo diferença significativa apenas nas doenças crônicas do parênquima hepático. ${ }^{11}$ No momento final deste protocolo, não houve variação significativa destes valores entre os momentos experimentais (Tabela 1), sendo que houve tendência à diminuição destas médias o que está de acordo com o estágio agudo do quadro clínico instalado.

O processo inflamatório composto por células mononucleares e polimorfonucleares ou ambas as células, foi observado pelos exames citopatológicos e histopatológico em todos os animais avaliados ao final do experimento. Adicionalmente, um animal apresentou achados compatíveis com esteatose, observado pela presença de macrovacúolos citoplasmáticos dos hepatócitos. Em protocolo semelhante ${ }^{9}$, todos os animais apresentaram algum tipo de infiltrado mononuclear ou polimorfonuclear e microvacuolização citoplasmática, caracterizando inflamação do parênquima hepático.

No presente estudo, não foi possível caracterizar diferença estatística nos valores das variáveis estudadas nos diferentes momentos experimentais. Ressaltamos a importância da padronização dos valores do NMOST/ NALL, LMEAN e SD para melhor avaliação de variações da ecogenicidade e ecotextura do fígado de gatos. De acordo com os dados obtidos neste estudo, a avaliação ultrassonográfica, pela função histograma dos níveis de cinza, é uma técnica que permitirá a caracterização da ecogenicidade e ecotextura do fígado de gatos e de outras espécies, possibilitando diagnóstico mais precoce e com precisão das alterações difusas do parênquima hepático, mas merecendo mais estudos.

A tirotoxicose gerou um quadro 
compatível de hepatite tirotóxica verificada por meio da citopatologia e histopatologia, porém ao exame ultrassonográfico quantitativo não foram encontradas alterações que indicassem insultos ao fígado desses animais. Entretanto, durante o período experimental de sessenta dias, a tirotoxicose não foi capaz de promover repercussão clínica nos animais do grupo experimental. Concomitante a este estudo, foram avaliados outros sistemas orgânicos quanto aos efeitos da tirotoxicose em gatos, e serão descritos em outros manuscritos.

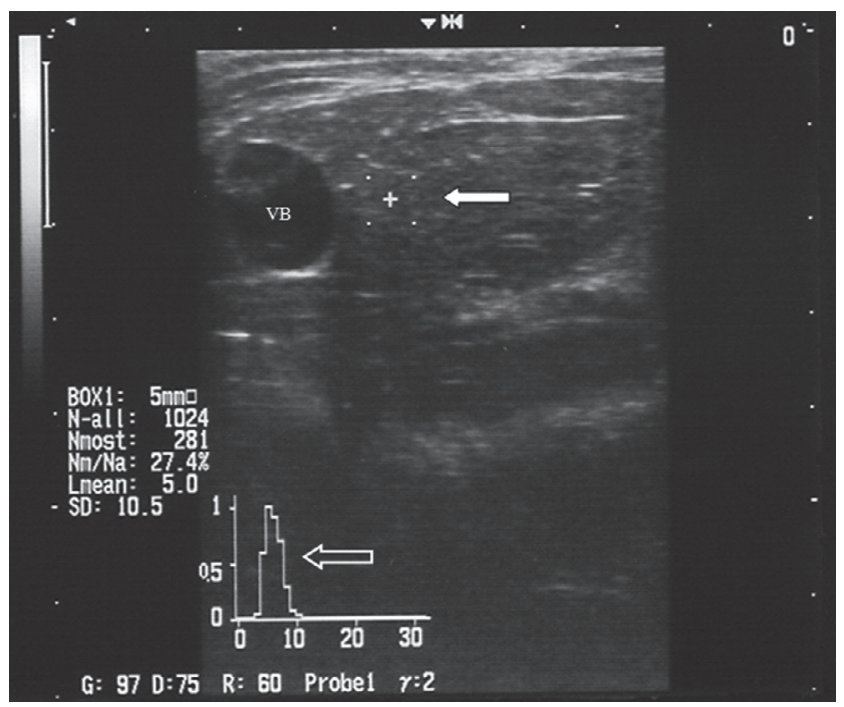

Figura 1 - Imagem ultrassonográfica quantitativa do fígado de um gato antes do início do protocolo de indução à tirotoxicose. A área quadrangular lateral à vesícula biliar (seta cheia) selecionada para mensuração da ecotextura e ecogenicidade do parênquima hepático. VB: vesícula biliar. Seta vazia indicando o gráfico do histograma dos níveis de cinza

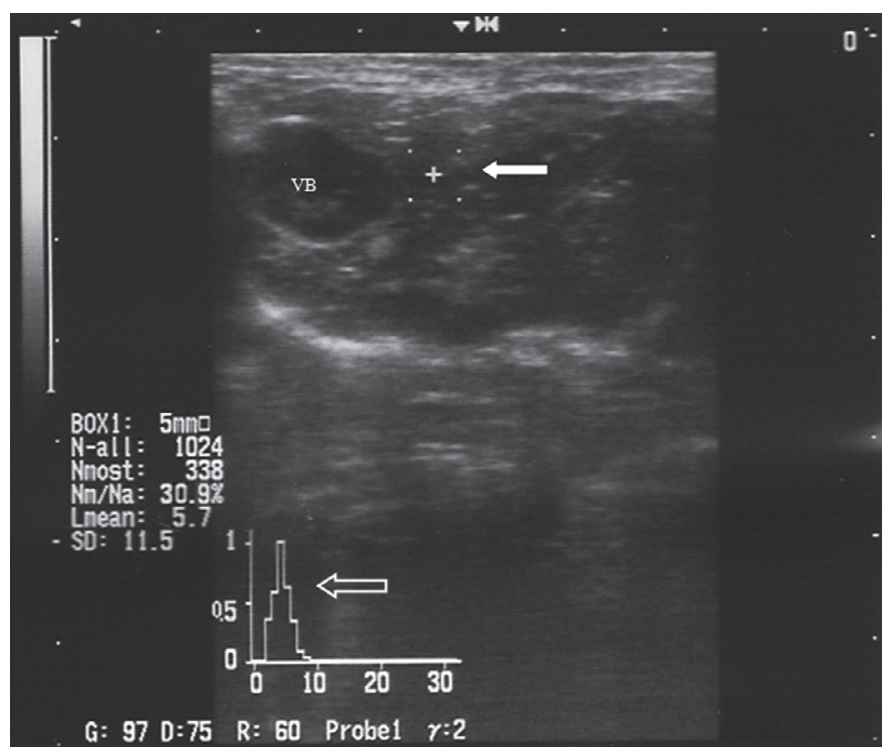

Figura 2 - Imagem ultrassonográfica quantitativa do fígado de um gato após término do protocolo de indução à tirotoxicose. A área quadrangular lateral à vesícula biliar (seta cheia) selecionada para mensuração da ecotextura e ecogenicidade do parênquima hepático. VB: vesícula biliar. Seta vazia indicando o gráfico do histograma dos níveis de cinza 


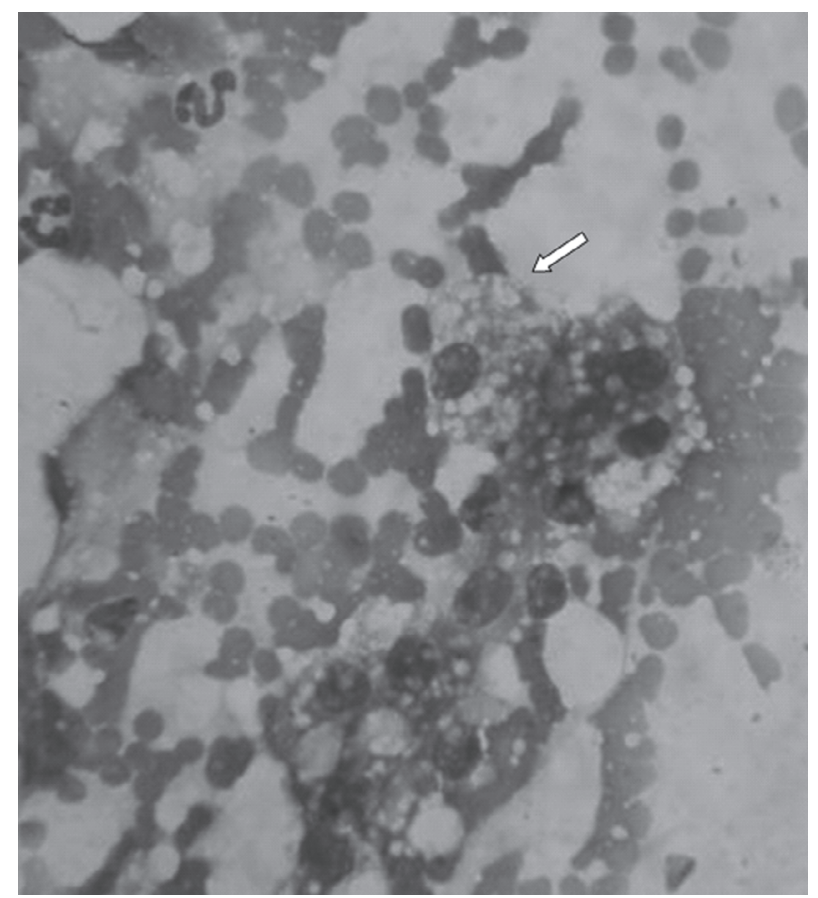

Figura 3 - Fotomicrografia de amostra citopatológica do fígado de gato com tirotoxicose experimental demonstrando inúmeros hepatócitos com intensa vacuolização citoplasmática (seta). Objetiva 100x, coloração Panótico rápido

\title{
Quantitative ultrasond of the liver in cats with induced thyrotoxicosis
}

\begin{abstract}
This study evaluated ultrasonographically the liver of cats submitted to induced thyrotoxicosis by the technique of gray-level histogram with the objective of better evaluating the possible hepatic alterations decurring from the excess of the thyroid hormones. For that, there were used nine adult-young healthy cats, which received daily 150ìg/ $\mathrm{kg}$ of sodic levotiroxin, for 60 days. The hepatic histogram has not demonstrated statistical differences in the $5 \%$ level of significance between the evaluated moments; however, the variations of the studied variables' values suggested an increasing of the heterogeneity and a decreasing of the echogenicity of the hepatic parenchyma when compared to the initial moment. The cytopathologic and histopathologic evaluations have shown of a clinical picture of thyrotoxic hepatitis in all the animals.
\end{abstract}

\section{Referências}

1 MOONEY, C. T. Hyperthyroidism. In: ETTINGER, S. J.; FELDMAN, E. C. (Ed.). Textbook of veterinary internal medicine. 6. ed. St. Louis: Elsevier Saunders, 2005. v. 2, p. 1544-1560.

2 PETERSON, M. E. Diagnostic tests for hyperthyroidism in cats. Clinical Techniques in Small Animal Practice,
Key words: Thyrotoxicosis.

Liver.

Ultrasound. Histogram. Cats v. 21, n. 1 , p. $2-9,2006$

3 HUANG, M. J.; LIAW, Y. F. Clinical associations between thyroidand liver diseases. Journal of Gastroenterology and Hepatology, v. 10, n. 2, p. 344350, 1995.

4 GILMAN, A. G.; MURAD, F. Thyroid and antithyroid drugs. In: GOODMAN, L. C.; GILMAN, A. The pharmacological basis of therapeutics. 5. ed. New York: 
MacMilliam, 1975. p. 1398-1422.

5 SOUZA, H. J. M. Particularidades da terapêutica. In: SOUZA, H. J. M. (Org.). Coletâneas em medicina e cirurgia de felinos. Rio de Janeiro: L. F. Livros de Veterinária, 2003. p. 349-361.

6 BROUSSARD, J. D.; PETERSON, M. E.; FOX, P. R. Changes in clinical and laboratory findings in cats with hyperthyroidism from 1983 to 1993. Journal of the American Veterinary Medical Association, v. 206, n. 3, p. 302-305, 1995.

7 VIDELA, L. A.; SMOKT, G.; TRONCOSO, P.; SIMON, K. A.; JUNQUEIRA, V. B. C.; FERNÁNDEZ, $\mathrm{V}$. Influence of hyperthyroidism on lindane-induced hepatotoxicity in the rat. Biochemical Pharmacology, v. 50, n. 10, p. 1557-65, 1995.

8 ENGELMAN, M. F. B.; GUOIDUGLI NETO, J.; ANDRADE, C. H. V.; HERNANDEZ, R.; GOULART, L. B. N. T. Estudo morfométrico do fígado de ratos submetidos a doses supra-fisiológicas de tiroxina. Arquivos Brasileiros de Endocrinologia \& Metabologia, v. 45, n. 2, p. 173-179, 2001.

9 ZABLITH, A. C. A. Tirotoxicose experimental em gatos: estudo ultra-sonográfico das alterações hepáticas e suas correlações com os níveis séricos das enzimas hepáticas dos hormônios tireóideos e achados histológicos e citológicos. 2004. 77 f. Dissertação (Mestrado) - Faculdade de Medicina Veterinária, Universidade Estadual Paulista "Júlio de Mesquita Filho", Botucatu, São Paulo, 2004.

10 TCHELEPI, H.; RALES, P. W.; RADIN, R. W. GRANT, E. Sonography of difuse liver disease. Journal of Ultrasound in Medicine, v. 21, n. 9, p. 1023-1032, 2002.

11 LEE, H. C.; CHOI, J. W.; KIM, K. A.; SEO, T. S.; LEE, J. M.; PARK, C. M. Usefulness of standard deviation on the histogram of ultrasound as a quantitative value for hepatic parenchymal echo texture; preliminary study. Ultrasound in Medicine \& Biology, v. 32, n. 12, p. 1817-1826, 2006.

12 BILLER, D. S.; KANTROWITZ, B.; MIYABAYASHI, T. Ultrasonography of diffuse liver disease. Journal of Veterinary Internal Medicine, v. 6, n. 2, p. 71-76, 1992.

13 NICOLL, R. G.; O'BRIEN; R. T.; JACKSON, M. W. Qualitative ultrasonography of the liver in obese cats. Veterinary Radiology \& Ultrasound, v. 39, n. 1, p. 4750, 1998.

14 PARTINGTON, B. P.; BILLER, D. S. Hepatic imaging with radiology and ultrasound. Veterinary Clinics of North America: Small Animal Practice, v. 25, n. 2, p. 305-335, 1995

15 ZWEIBEL. W. J. Sonographic diagnosis of diffuse liver disease. Seminars in Ultrasound, CT and MRI, v. 16, n. 11, p. 8-15, 1995.

16 NYLAND, T. G.; MATTOON, J. S.; HERRGESELL, E. J.; WISNER, E. R. Fígado. In: NYLAND, T. G.;
MATOON, J. S. Ultra-som: diagnóstico em pequenos animais. 2. ed. São Paulo: Roca, 2004. p. 95-127.

17 OSAWA, H.; MORI, Y. Sonographic diagnosis of fatty liver using a histogram technique that compares liver and renal cortical echo amplitudes. Journal of Clinical Ultrasound, v. 24, n. 1, p. 25-29, 1996.

18 LU, Z. F.; ZAGZEBSKI, J. A.; O'BRIEN, R. T.; STEINBERG, $\mathrm{H}$. Ultrasound attenuation and backscatter in the liver during prednisone administration. Ultrasound in Medicine \& Biology, v. 23, n. 1, p. 1-8, 1997.

19 MAEDA, K.; UTSU, M.; KIHAILE, P. E. Quantification of sonographic echogenicity with greylevel histogram width: a clinical tissue characterization. Ultrasound in Medicine \& Biology, v. 24, n. 2, p. 225234, 1998.

20 NICOLL, R. G.; JACKSON, M. W.; KNIPP, B. S.; ZAGZEBSKI, J. A.; STEINBERG, H.; O'BRIEN, R. T. Quantitative ultrasonography of the liver in cats during obesity induction and dietary restriction. Research in Veterinary Science, v. 64, n. 1, p. 1-6, 1998.

21 FALLON, M. D.; PERRY, H. M.; BERGFELD, M.; DROKE, D.; TEITELBAUM, S. L.; AVIOLI, L. V. Exogenous hyperthyroidism with osteoporosis. Archives of Internal Medicine, v. 143, n. 3, p. 442-444, 1983.

22 PIATNEK-LEUNISSEN, D.; OLSON, R. E. Cardiac failure in the dog as a consequence of exogenous hyperthyroidism. Circulation Research, v. 20, n. 2, p. 242-252, 1967.

23 MUNDY, G. R.; SHAPIRO, J. L.; BANDELIN, J. G.; CANALIS, E. M.; RAISZ, L. G. Direct stimulation of bone resorption by thyroid hormones. Journal of Clinical Investigation, v. 58, n. 3, p. 529-534, 1976.

24 KUNG, A. W.; NG, F. A rat model of thyroid hormone-induced bone loss: effect of antiresorptive agents on regional bone density and osteocalcin gene expression. Thyroid, v. 4, n. 1, p. 93-98, 1994.

25 SERAKIDES, R.; NUNES, V. A.; NASCIMENTO, E. F.; RIBEIRO, A. F. C.; ZIBAOUI, H. M.; SILVA, C. M Relação tireóide-gônadas e níveis plasmáticos de fósforo, cálcio e fosfatase alcalina em ratas. Arquivo Brasileiro de Medicina Veterinária e Zootecnia, v. 52, n. 6, p. 579-585, 2000.

26 SERAKIDES, R.; NUNES, V. A.; NASCIMENTO, E. F.; RIBEIRO, A. F. C.; ZIBAOUI, H. M.; SILVA, C. M. Hipogonadismo e função tireoidiana em ratas hipertireóideas e eutireóideas. Arquivo Brasileiro de Medicina Veterinária e Zootecnia, v. 52, n. 6, p. 571578, 2000.

27 BABINSKI, L. K.; HAENISCH, S. L.; COSTA, F. S.; CARDOSO, M. J. L.; MUNIZ, M. J. L.; LAMBERTI, D. G. Avaliação radiográfica do eixo ápico-basilar do coração em gatos (Felis catus, L. 1758) após tirotoxicose induzida. In: CONGRESSO INTERNACIONAL DE MEDICINA FELINA, 2., 2001, Rio de Janeiro. Anais... Rio de Janeiro: 2001. p. 37-38. 
28 COSTA, F. S. Tirotoxicose experimental em gatos: efeitos sobre o tecido ósseo, níveis séricos de fosfatase alcalina e metabolismo de cálcio e fósforo. 2002. 101 f. Dissertação (Mestrado) - Faculdade de Medicina Veterinária, Universidade Estadual Paulista "Júlio de Mesquita Filho", Botucatu, São Paulo, 2002.

29 COSTA, F. S.; CARDOSO, M. J. L.; MUNIZ, L. M. R.; VULCANO, L. C.; PADOVANI, C. R. Avaliação da densidade mineral óssea em gatas castradas após tirotoxicose induzida. Veterinária Ser, v. 1, n. 2, p. 104-107, 2004.

30 COSTA, F. S.; CARDOSO, M. J. L.; MUNIZ, L. M. R.; VULCANO, L. C.; PADOVANI, C. R. Determinação de desmineralização óssea em gatos após tirotoxicose experimental. Brazilian Journal of Veterinary Research and Animal Science, v. 43, n. 5, p. 695-701, 2006.

31 LUKENSMEYER, W. W.; HEGE, J. H.; THEIL, G. B.; WILSON, W. R. Calcium and phosphorus metabolic studies in triiodothyronine-induced hypermetabolism. American Journal of the Medical Sciences, v. 259, n. 4, p. 282-291, 1970.

32 INTITUTO NACIONAL DE PESQUISAS ESPACIAIS. Divisão de processamento de imagens. (INPE, DPI). Processamento digital de imagens: estatísticas sobre imagens digitais. Disponível em: http:// www.dpi.inpe.br/ carlos/Academicos/Cursos/Pdi/ pdi_estatisticas.html >. Acesso em: 20 fev. 2009. 